DOI: $10.1111 / \mathrm{ijcp} .14550$

\title{
Multiple vertebral fractures after suspension of denosumab. A series of 56 cases
}

\author{
Manuel Sosa-Henríquez ${ }^{1}$ (i) | Oscar Torregrosa ${ }^{2}$ | Alejandro Déniz ${ }^{3}$ | Pedro Saavedra ${ }^{4}$ | \\ Norberto Ortego $^{5}$ | Ana Turrión ${ }^{6}$ | José Luis Pérez Castrillón ${ }^{7}$ | Manuel Díaz-Curiel ${ }^{8}$ | \\ Carlos Gómez-Alonso9 | Guillermo Martínez ${ }^{10}$ | José Antonio Blázquez ${ }^{11}$ | \\ José Manuel Olmos-Martínez ${ }^{12}$ | Î́nigo Etxebarria ${ }^{13}$ | José Ramón Caeiro ${ }^{14}$ | \\ Damián Mora-Peña ${ }^{15}$
}

\footnotetext{
${ }^{1}$ University of Las Palmas de Gran Canaria, Investigation Group on Osteoporosis and Bone Mineral Metabolism, Hospital University Insular, Las Palmas de Gran Canaria, Canary Islands, Spain

${ }^{2}$ Internal Medicine Service, Hospital General University Elche, Elche, Alicante, Spain

${ }^{3}$ Endocrinology Section, Hospital University Insular, Las Palmas de Gran Canaria, Spain

${ }^{4}$ Mathematics Department, University of Las Palmas de Gran Canaria, Las Palmas de Gran Canaria, Spain

${ }^{5}$ Internal Medicine, Hospital University San Cecilio, Granada, Spain

${ }^{6}$ Rheumatology Service, Hospital University Salamanca, Salamanca, Spain

${ }^{7}$ Internal Medicine, Hospital University Río Hortega, Valladolid, Spain

${ }^{8}$ Internal Medicine Service, Hospital University Fundación Jiménez Díaz, Madrid, Spain

${ }^{9}$ Internal Medicine Service, Hospital University Central Asturias, Oviedo, Spain

${ }^{10}$ Endocrinology Service, Hospital University 12 de Octubre, Madrid, Spain

${ }^{11}$ Internal Medicine Service, Hospital General University Albacete, Albacete, Spain

${ }^{12}$ Internal Medicine Service, Hospital Universitario Marqués de Valdecilla, Santander, Spain

${ }^{13}$ Orthopaedic Surgery, Hospital Alto Deba, Guipúzcoa, Spain

${ }^{14}$ Orthopaedic Surgery Department, University Hospital of Santiago de Compostela, La Coruña, Spain

${ }^{15}$ Internal Medicine Service, Hospital Virgen de la Luz, Cuenca, Spain
}

\section{Correspondence}

Manuel Sosa-Henríquez, University of Las Palmas de Gran Canaria, Investigation Group on Osteoporosis and Bone Mineral Metabolism, Hospital University Insular, Las Palmas de Gran Canaria, Canary Islands, Spain.

Email: manuel.sosa@ulpgc.es

\begin{abstract}
Background: Denosumab is a monoclonal antibody approved for the treatment of postmenopausal osteoporosis. The withdrawal of denosumab produces an abrupt loss of bone mineral density and may cause multiple vertebral fractures (MVF).

Objective: The objective of this study is to study the clinical, biochemical, and densitometric characteristics in a large series of postmenopausal women who suffered MVF after denosumab withdrawal. Likewise, we try to identify those factors related to the presence of a greater number of vertebral fractures (VF).

Patients and Methods: Fifty-six patients ( 54 women) who suffered MVF after receiving denosumab at least for three consecutive years and abruptly suspended it. A clinical examination was carried out. Biochemical bone remodelling markers (BBRM) and bone densitometry at the lumbar spine and proximal femur were measured. VF
\end{abstract}


were diagnosed by magnetic resonance imaging MRI, X-ray, or both at dorsal and lumbar spine.

Results: Fifty-six patients presented a total of 192 VF. 41 patients (73.2\%) had not previously suffered VF. After discontinuation of the drug, a statistically significant increase in the BBRM was observed. In the multivariate analysis, only the time that denosumab was previously received was associated with the presence of a greater number of VF $(P=.04)$.

Conclusions: We present the series with the largest number of patients collected to date. 56 patients accumulated 192 new VF. After the suspension of denosumab and the production of MVF, there was an increase in the serum values of the BBRM. The time of denosumab use was the only parameter associated with a greater number of fractures.

\section{1 | INTRODUCTION}

Denosumab (DMAB), a monoclonal antibody against the receptor activator of nuclear factor k-B ligand (RANKL), is a potent antiresorptive agent commonly prescribed in patients with postmenopausal osteoporosis. DMAB reduces bone resorption and improves bone mineral density (BMD). ${ }^{1}$ The FREEDOM trial found reduced risk of fragility fracture, a study that lasted 10 years. ${ }^{2,3}$

Unlike bisphosphonates, which have a residual effect on bone when deposited therein, ${ }^{4}$ discontinuing DMAB treatment may produce a rebound effect on markers of bone remodelling and a loss of bone mass to the extreme that their values are even below the existing values before starting treatment. ${ }^{5}$ Furthermore, since 2015 , several case reports and series were published describing multiple vertebral fractures (MVF) in patients discontinuing DMAB, which are also characterised by being painful. ${ }^{6-9}$ Recently, three cases have been described of patients who suffered a hip fracture after the suspension of DMAB ${ }^{10}$ and also repeated fractures in the same patient. ${ }^{11}$ The mechanism by which this complication occurs is unknown, as is its exact incidence. ${ }^{11}$

Most of the articles published to date describe isolated cases or series with few patients. In this study, we present a series of 56 patients who suffered multiple vertebral fractures after discontinuing $D M A B$ and a study of their clinical, analytical, and densitometric characteristics. This series includes the largest number of patients published so far, with the aim of identifying prognostic factors for higher risk patients and establish the most appropriate preventive actions.

\section{PATIENTS AND METHODS}

\section{1 | Selection of the patients. clinical data acquisition}

The study was carried out in Spain, between 1 April 2019 and 31 January 2020, coordinated by the working group on osteoporosis and mineral metabolism of the Spanish Society of Internal Medicine (SEMI).

\section{What's known}

- Denosumab is a drug used in the treatment of osteoporosis.

- When denosumab is stopped, a rebound effect may appear that leads to a massive bone loss and the development of multiple vertebral fractures.

- This side effect is little known.

\section{What's new}

- Now, we know that bone remodelling is increased when the drug is stopped.

- Denosumab use was the only parameter associated with a greater number of fractures.

- The more years denosumab is used, the greater the risk of vertebral fractures when it is stopped.

Patients who had previously received a minimum of 1 year of DMAB treatment, injecting at least two doses, having produced a minimum delay of 2 months from the moment of injection, were included.

Each patient completed a questionnaire recording demographic data, body mass index (BMI), and risk factors for osteoporosis. We also calculated the risk of suffering a major osteoporotic fracture (MOF) and specifically a hip fracture (HF) using the FRAX ${ }^{\circledR}$ tool. Xrays from the spine were carried out in all patients before starting the treatment with DMAB with the exception of those patients who had a recent $X$-ray taken in the three months before the start of the treatment.

\section{2 | Vertebral fracture diagnosis}

Patients must have suffered at least one fragility fracture after discontinuation of DMAB. This fracture was verified by a lateral 
radiography of the thoracic and lumbar spine, an magnetic resonanceimaging (MRI) of the entire spine, or both. All the fractures were symptomatic so the MRI or X-rays were done at the moment the patient reported the back pain.

All patients had at least one X-ray study before the start of the study. Vertebral fracture diagnosis was confirmed by MRI assessed by a radiologist, except in four patients in which it was based on shape changes in X-ray exams as compared with recent previous images. Genant's classification ${ }^{12}$ was applied to diagnose vertebral fracture. Those patients with cancer, Paget's disease of bone, or when the fracture was traumatic were excluded.

\section{3 | Bone mineral densitometry}

All patients had at least two dual X-ray absorptiometry (DXA) exams: one before or at the time of DMAB initiation and one after vertebral fracture occurrence. Exams were carried out with different machines for different patients, but the same for each patient, allowing us to compare both exams. Because of the presence of several vertebral fractures in all the patients, bone mineral measurement at the lumbar spine was excluded. T-scores at the hip were calculated using normal values for the Spanish population. For biochemical determinations, fasting blood was drawn. The biochemical parameters, creatinine, total proteins, calcium, and phosphorus, were measured using standardised colorimetric methods. Immunochemiluminescence was used to determine the biochemical parameters of bone remodelling: P1NP, beta-crosslaps, and osteocalcin.

The study was carried out following the rules of the Declaration of Helsinki, ${ }^{13}$ the protocol approved by the Insular University Hospital of Gran Canaria Clinical Trials Committee. All patients were informed of the study objectives and gave their informed written consent.

\section{3 | STATISTICAL ANALYSIS}

\section{1 | Univariate analysis}

Categorical variables are expressed as frequencies and percentages and continuous as mean and standard deviation (SD). Paired means were compared using the Wilcoxon test for paired data.

\subsection{Poisson models}

The effect of each factor $(X)$ on the number of vertebral fractures after DMAB (nVF) was analysed by means of the Poisson model: nVFPoisson $(\mu)$, being

$$
\log (\mu)=\alpha+\beta X
$$

where $\mu$ is the expected number of vertebral fractures, which may depend on the $X$ factor. When $X$ is a binary variable indicating presence or absence of a character, its values were coded as 1 (presence) and 0 (absence). From this model, it follows

$$
\frac{\mu(X=t+1)}{\mu(X=t)}=\exp (\beta)
$$

where $\mu(X=t)$ corresponds to the expected number of vertebral fractures when the factor $X$ is in level t. Therefore, $\exp (\beta)$ correspond to the proportion of variation of the expected number of vertebral fractures for each unit that varies $X$.

Statistical significance was set at $P<.05$. Data were analysed using the $\mathrm{R}$ package, version 3.6.1 ( $\mathrm{R}$ Development Core Team, 2019).

\section{4 | RESULTS}

Table 1 shows the baseline characteristics of our study patients. A total of 56 patients were included, of which 54 were women (96.4\%). The mean age was $68.1 \pm 8.2$ years. The most frequently observed concomitant diseases were arterial hypertension (32.1\%), dyslipidemia (32.1\%), and hypothyroidism (16.1\%). Most of the patients had not previously suffered vertebral fractures (73.2\%), and their risk of fracture calculated at 10 years using the FRAX risk assessment tool after having suffered multiple vertebral fractures was $11 \%$ for major fractures (95\% confidence interval $[\mathrm{Cl}] 6.1-16 \%)$ and $3.9 \%$ for hip

\begin{tabular}{|c|c|}
\hline & Media \pm SD \\
\hline Number & 56 \\
\hline Age (y) & $68.1 \pm 8.2$ \\
\hline Weight (kg) & $60.7 \pm 12.3$ \\
\hline Height (m) & $1.6 \pm 0.1$ \\
\hline \multirow[t]{2}{*}{$\mathrm{BMI}\left(\mathrm{kg} / \mathrm{m}^{2}\right)$} & $25.1 \pm 4.6$ \\
\hline & Number (\%) \\
\hline Sex female & $54(96.4)$ \\
\hline Diabetes mellitus & $3(5.4)$ \\
\hline Arterial hypertension & $18(32.1)$ \\
\hline Dyslipidemia & $18(32.1)$ \\
\hline Hypothyroidism & $9(16.1)$ \\
\hline Concomitant use of calcium and vitamin D & $41(73.2)$ \\
\hline \multicolumn{2}{|c|}{$\begin{array}{l}\text { Prevalence of fractures before the appearance of multiple vertebral } \\
\text { fractures }\end{array}$} \\
\hline No vertebral fracture & $41(73.2)$ \\
\hline One vertebral fracture & $4(7.1)$ \\
\hline Two vertebral fractures & $2(3.6)$ \\
\hline Three vertebral fractures & $1(1.8)$ \\
\hline Four vertebral fractures & $2(3.6)$ \\
\hline Nonvertebral fractures & $5(8.9)$ \\
\hline Hip fracture & $1(1.8)$ \\
\hline
\end{tabular}

TABLE 1 Characteristics of the population studied

Abbreviation: SD, standard deviation. 


\begin{tabular}{ll|} 
& Median $\mathrm{Cl} 95 \%$ \\
\hline FRAX (Major) & $11.0(6.1-16.0)$ \\
\hline FRAX (Hip) & $3.9(1.2-6.6)$ \\
\hline Time using denosumab (months) & $30.5(24.0-43.5)$ \\
\hline Number of dose $(\mathrm{n})$ & $6.0(4.0-8.0)$ \\
\hline $\begin{array}{l}\text { Time after last dose of denosumab and multiple vertebral fractures } \\
\text { (months) }\end{array}$ & $11.0(7.5-13.5)$ \\
\hline Number of vertebral fractures after denosumab withdrawal $(\mathrm{n})$ & $3(2-4)$ \\
\hline Number of vertebral fractures accumulated $(\mathrm{n})$ & 192 \\
\hline
\end{tabular}

TABLE 2 Ten-year risk of fracture (FRAX) in percentage after the appearance of multiple vertebral fractures, number of fractures per patient, and total accumulated

Data related to the use and withdrawal of denosumab (time using denosumab, number of dose, and time after last dose of denosumab).

Abbreviation: $\mathrm{Cl}$, confidence interval.

fractures (1.2-6.6\%). Patients had been taking DMAB for a median of 30.5 months ( $95 \% \mathrm{Cl}$ : 24-43.5 months) and had injected a median of six doses ( $95 \% \mathrm{Cl}$ : 4-8 doses). 56 patients accumulated 192 new vertebral fractures.

Table 2 shows the 10-year risk of fracture (FRAX) in percentage after the appearance of multiple vertebral fractures, number of fractures per patient, and total accumulated data related to the use and withdrawal of DMAB (time using DMAB, number of dose, and time after last dose of DMAB). Most patients were considered as high risk for hip fracture (considered as a 10 -year risk higher than $3 \%$ ) but not for major fracture which is considered a 10-year risk higher than $20 \%$. The median dose received was six, and the median of vertebral fractures that suffered each patient was three. The total number of accumulated vertebral fractures was 192 .

Table 3 shows the reasons DMAB was discontinued. Medical prescription was the main cause of suspending treatment, which occurred in 23 patients (41.1\%). In 12 cases (21.5\%), the discontinuation was ordered by an odontologist in order to carry out a buccal procedure. In 15 patients (26.7\%), the decision to discontinue DMAB was taken by the patients themselves, sometimes because they forgot to administer the drug, due to adverse effects such as fatigue or other secondary effects. Finally, there were six patients (10.7\%) with other reasons for stopping the treatment with $\mathrm{DMAB}$, such as economic reasons (they could not afford the drug) or difficulty to find someone to administer the DMAB in times of pandemic confinement.

Table 4 shows the biochemical values studied, including the biochemical markers of bone remodelling, obtained before and after DMAB suspension, and the appearance of multiple vertebral fractures. Values of calcium, phosphorus, total proteins, vitamin D (25 hydroxycholecalciferol), and parathyroid hormone (PTH) do not change substantially, but the biochemical markers of bone remodelling increase significantly, both beta-crosslaps, P1NP, and osteocalcin ( $P<.006$ in all cases). The greatest increase occurs in the beta-crosslaps, from 0.071 to $0.520 \mathrm{ng} / \mathrm{mL}$ median, a 14 -fold increase in baseline values. Osteocalcin values almost tripled whereas those of P1NP quadrupled.

Finally, Table 5 shows the logistic regression analysis to study the possible association between the various clinical, analytical, and densitometric parameters and the number of vertebral fractures.
TABLE 3 Reason for denosumab withdrawal

\begin{tabular}{|lc|}
\hline Reason & $\begin{array}{l}\text { Number } \\
(\%)\end{array}$ \\
\hline Medical recommendation & $23(41.1)$ \\
\hline Side effects: osteomuscular pain & $10(17.9)$ \\
\hline Drug holidays or treat to target & $6(10.7)$ \\
\hline Not specified & $5(8.9)$ \\
\hline Cataracts & $1(1.8)$ \\
\hline Primary normocalcaemic hyperparathyroidism & $1(1.8)$ \\
\hline Dentist recommendation & $12(21.5)$ \\
\hline Patient's decision & $15(26.7)$ \\
\hline Oversight, forgotten & $5(8.9)$ \\
\hline Fatigue & $5(8.9)$ \\
\hline Secondary effects & $5(8.9)$ \\
\hline Others & $6(10.7)$ \\
\hline
\end{tabular}

The only factor associated with the presence of multiple vertebral fractures was the previous use of DMAB. For each year using $D M A B$, the risk of suffering new vertebral fractures was $11 \%$.

\section{5 | DISCUSSION}

Our study included a total of 56 patients and constitutes the largest number of cases collected in a single series. Previous studies presented a smaller number of cases. González-Rodríguez et al ${ }^{7}$ collected 60 spontaneous vertebral fractures in 15 women with breast cancer who were undergoing treatment with aromatase inhibitors and in whom DMAB was discontinued. Fernández Fernández et al ${ }^{14}$ described 49 vertebral fractures in 10 women, and Florez et al ${ }^{15}$ published a series of seven women who had a median of five vertebral fractures. Another study collected the first three cases of hip fracture produced after abrupt DMAB discontinuation in the absence of other causes. ${ }^{10}$ Several systematic reviews have confirmed the magnitude of the problem. ${ }^{6,16-18}$ In this series, we publish the first two cases described in men. 
TABLE 4 Biochemical parameters including bone remodelling markers before and after denosumab withdrawal and the appearance of multiple vertebral fractures

\begin{tabular}{|c|c|c|c|c|}
\hline & Prewithdrawal & Postwithdrawal & $P$-value ${ }^{a}$ & Percentage change \\
\hline Calcium (mg/dL) & $9.5(9.2 ; 9.8)$ & $9.7(9.2 ; 10.0)$ & .325 & $1.88(-3.42 ; 6.54)$ \\
\hline Phosphorus (mg/dL) & $3.50(3.12 ; 3.77)$ & $3.60(3.25 ; 3.85)$ & .491 & $2.33(-11.11 ; 11.21)$ \\
\hline Total proteins (g/L) & $7.1(6.9 ; 7.3)$ & $7.0(6.6 ; 7.2)$ & .410 & $-2.34(-5.41 ; 2.90)$ \\
\hline Beta-crosslaps (ng/mL) & $0.07(0.05 ; 0.31)$ & $0.52(0.44 ; 1.09)$ & $<.001$ & $1,367(110 ; 1,866)$ \\
\hline Osteocalcin (ng/mL) & $10.7(8.38 ; 14.1)$ & $28.1(21.4 ; 33.0)$ & .125 & $196(140 ; 243)$ \\
\hline Vitamin $D^{c}(\mathrm{ng} / \mathrm{mL})$ & $29.7(25.9 ; 39.8)$ & $31.0(26.4 ; 44.8)$ & .438 & $30.5(-12.8 ; 103.5)$ \\
\hline PTH (pg/mL) & $50.1(39.0 ; 60.0)$ & $46.8(36.6 ; 56.2)$ & .875 & $7.02(-17.52 ; 34.94)$ \\
\hline TS-spine & $-2.19(-2.86 ;-1.35)$ & $-2.08(-2.88 ;-1.16)$ & .739 & $-0.67(-56.80 ; 17.69)$ \\
\hline
\end{tabular}

Data are medians (interquartil range). PTH, parathyroid hormone.

${ }^{a}$ Wilcoxon test for paired data.

${ }^{\text {b}}$ Type I procolagen amino-terminal peptide.

'25 hydroxicholecalciferol (25-HCC).

TABLE 5 Association of the number of vertebral fractures with each one of the factors shown, adjusted by age ${ }^{a}$

\begin{tabular}{lll}
\hline Factor & Relative risk $(95 \% \mathrm{CI})$ & $P$-value \\
\hline Time using denosumab, per each year & $1.110(1.005-1.226)$ & .044 \\
\hline Diabetes mellitus & $0.701(0.319-1.541)$ & .381 \\
\hline Arterial hypertension & $0.856(0.613-1.193)$ & .362 \\
\hline Dyslipidemia & $0.794(0.574-1.098)$ & .169 \\
\hline Hypothyroidism & $1.054(0.708-1.569)$ & .795 \\
\hline DXA lumbar spine-before & $2.546(0.578-11.221)$ & .225 \\
\hline DXA lumbar spine-post & $0.348(0.087-1.394)$ & .144 \\
\hline Creatinine-post & $0.617(0.234-1.623)$ & .333 \\
\hline Calcium-post & $0.970(0.900-1.046)$ & .433 \\
\hline Phosphorus-post & $0.973(0.809-1.171)$ & .775 \\
\hline Total proteins-post & $0.862(0.598-1.243)$ & .431 \\
\hline
\end{tabular}

Abbreviation: $\mathrm{Cl}$, confidence interval.

${ }^{a}$ Each relative risk was obtained by means of a Poisson regression, being the dependent variable the number of vertebral fractures and the covariates, the corresponding factor and the age.
The actual number of cases is probably much higher. The Spanish Agency for Medicines and Health Products (AEMPS), which collects adverse effects of drugs, described in 2019 a total of 64 patients with multiple vertebral fractures that were increased in a subsequent statement in 2020, 213 patients with multiple vertebral fractures, and 50 hip fractures. There are several reasons that might explain why the magnitude of the problem is not perceived. We would mention the following: (a) it is a complication not yet sufficiently known by the medical community in general; (b) they are fractures that occur in patients who have osteoporosis, therefore, they can be attributed to the disease rather than to the suspension of the drug; (c) given that the drug is administered every 6 months, it is possible to forget it, especially when the questioning is directed at drugs that are taken orally; and (d) for scientific journals, the publication of new cases does not provide anything noteworthy. So, in recent years, the number of publications on the matter has decreased, and the number of fractures has not.

The mean age of our series was 68.1 years, somewhat older than those described in other series, such as that of Barcelona, where the median age was 65 years, ${ }^{15}$ and that of Madrid with a mean of 66.4 years. ${ }^{14}$ In the González-Rodríguez series, ${ }^{7}$ the mean age was lower, 62.3 years, but they were other types of patients, women with breast cancer and not postmenopausal osteoporosis. In a systematic review in which 24 cases were collected, the mean age was 64.1 years. $^{6}$ 
The reasons why DMAB was stopped varied considerably. Most of the withdrawals $(41.1 \%)$ were indicated by a doctor because of the development of side effects, mainly osteo-muscular pain. In 12 patients (21.5\%), the drug was discontinued by an odontologist in order to perform a buccal procedure and to avoid the risk of developing an osteonecrosis of the jaws. There were many other reasons. In 15 cases (26.7\%) was the patient's decision to stop the treatment because of side effect, without consulting the doctor. Finally, the economic crisis was another reason, because in some other patients, included as others, the patients could not afford the price of DMAB.

Our patients had received a median of six doses, with DMAB having been used a median of 30.5 months. These results coincide with those published in other series and reports of individual cases. ${ }^{6-10,14-16,19-22}$ In a "real world" study, the risk of fracture when discontinuing DMAB treatment has been calculated to increase markedly when the third injection is given. ${ }^{16}$ The time it takes for fractures to occur after the last dose of DMAB showed a median of 11 months in our study, which represents a 5-month delay, since the drug is administered every 6 months, although in one case, it occurred after the delay of a month and a half. In different reported cases, this period ranges from 2 to 13 months. ${ }^{6,8,14,15}$

Probably, the appearance of fractures will depend on two factors, the severity of the disease and the withdrawal of the drug. The severity of the disease could be determined through the FRAX or by the presence of previous fractures. The 10-year risk of fracture calculated by the FRAX tool showed a median of $11 \%$ for major fracture and $3.9 \%$ for hip fracture. Although there is a debate on the optimal threshold to perform a therapeutic intervention, ${ }^{23-25}$ the high risk of fracture has been established at $20 \%$ for the major fracture and $3 \%$ for the hip fracture. ${ }^{26}$ In our study, the fracture risk at 10 years showed a median of $11 \%$ for the major fracture and $3.9 \%$ for the hip fracture. FRAX has rarely been estimated in the publications of other cases.

The other factor involved is the discontinuation of the drug. One of the reasons DMAB was discontinued came about after reported improvement in treatment with BMD, leading to the misconception that osteoporosis was cured. Following this line, the idea of the "treat to target" was developed according to which, when reaching a certain $T$-score value, the drug could be suspended, without verifying the results of this suspension. ${ }^{27-29}$ This led to the discontinuation of DMAB due to medical recommendation in $41.1 \%$ of cases. Closely related to this idea is the concept of therapeutic holidays wrongly applied to DMAB. ${ }^{30,31}$ On the other hand, given that the association between the use of DMAB and osteonecrosis of the jaws has been described, ${ }^{32-34}$ the suspension of DMAB was carried out by the dentist's indication in $21.5 \%$ of the patients. Our results coincide with those reported in other series. ${ }^{7,8,22}$

The deleterious effect of DMAB suppression is determined by the sudden increase in remodelling that can lead to a deterioration in bone strength and facilitate the appearance of fractures. This fact had been previously described, although an increase in fractures had not been observed. After discontinuing DMAB, betacrosslaps increase significantly, from a median of 0.071 to $0.520 \mathrm{ng} /$
$\mathrm{ml}(P<.001)$. To a lesser extent but also significantly, the markers of bone formation increase, the P1NP that goes from 25.3 to $101.2 \mathrm{ng} / \mathrm{mL}, P=.006$, and osteocalcin from 10.7 to $28.1 \mathrm{ng} / \mathrm{mL}$. This indicates an increase in all bone remodelling in which osteoclastic activity clearly predominates, as has also been described in other series. ${ }^{20,35,36}$ We have not observed changes in serum levels of creatinine, calcium, phosphorus, total protein, vitamin D, measured as 25-hydroxyvitamin D, or in PTH.

Finally, we carried out a logistic regression analysis to try to identify which factors could be associated with the presence of a greater number of fractures, obtaining only a statistically significant association with the time in which DMAB was previously used $(P=.04)$. For each year using $D M A B$, the risk of suffering multiple vertebral fractures increased by $11 \%$. This is important because the more time the patient is receiving $D M A B$, the more the risk of developing multiple vertebral fractures, which is something that the doctors and patients should take into account when a new treatment with DMAB is indicated.

Among the limitation of our study is the sample size, which is due to the difficulty in identifying these patients. On the other hand, because there is no control group, we have not been able to establish what the clinical, analytical, or densitometric factors could be associated with the appearance of fractures. The strength of the study is determined by the high number of fractures associated with a full number of complementary tests.

To sum up, we present a series of 56 patients in which the abrupt discontinuation of DMAB caused a total of 192 vertebral fractures, the increase in bone removal probably being manifested through a considerable increase in biochemical markers of bone remodelling, especially those of resorption, which causes this effect.

\section{6 | ADDENDUM 1. OTHER RESEARCHERS}

Javier Martínez de Victoria Carazo, Daniel Fernández-Reyes, Carmen García-Ibarbia, Alejandro Domínguez-Regueiro, $M^{a}$ Jesús Moro-Álvarez, Miguel Ángel Mangas, Rafael Martín-Helguera, Javier del Pino-Montes, Olga Martínez, Miguel Ángel Tremiño-Arí, Diego Hernandez-Hernández.

\section{ORCID}

Manuel Sosa-Henríquez iD https://orcid.org/0000-0001-6845-2933

\section{REFERENCES}

1. Tsourdi E, Rachner TD, Rauner M, Hamann C, Hofbauer LC. Denosumab for bone diseases: Translating bone biology into targeted therapy. Eur J Endocrinol. 2011;165:833-840.

2. Cummings SR, Martin JS, McClung MR, et al. Denosumab for prevention of fractures in postmenopausal women with osteoporosis. N Engl J Med. 2009;361:756-765.

3. Ferrari S, Adachi JD, Lippuner $\mathrm{K}$, et al. Further reductions in nonvertebral fracture rate with long-term denosumab treatment in the FREEDOM open-label extension and influence of hip bone mineral density after 3 years. Osteoporos Int. 2015;26:2763-2771. 
4. Black DM, Reid IR, Cauley JA, et al. The effect of 6 versus 9 years of zoledronic acid treatment in osteoporosis: A randomized second extension to the HORIZON-pivotal fracture trial (PFT). J Bone Miner Res. 2015;30:934-944.

5. Popp AW, Varathan N, Buffat H, Senn C, Perrelet R, Lippuner K. Bone mineral density changes after 1 year of denosumab discontinuation in postmenopausal women with long-term denosumab treatment for osteoporosis. Calcif Tissue Int. 2018;103:50-54.

6. Anastasilakis AD, Polyzos SA, Makras P, Aubry-Rozier B, Kaouri S Lamy O. Clinical features of 24 patients with rebound-associated vertebral fractures after denosumab discontinuation: systematic review and additional cases. J Bone Miner Res. 2017;32:1291-1296.

7. Gonzalez-Rodriguez E, Aubry-Rozier B, Stoll D, Zaman K, Lamy O. Sixty spontaneous vertebral fractures after denosumab discontinuation in 15 women with early-stage breast cancer under aromatase inhibitors. Breast Cancer Res Treat. 2020;179:153-159.

8. Lamy O, Gonzalez-Rodriguez E, Stoll D, Hans D, Aubry-Rozier B. Severe rebound-associated vertebral fractures after denosumab discontinuation: 9 clinical cases report. J Clin Endocrinol Metab. 2017;102:354-358.

9. Tripto-Shkolnik L, Rouach V, Marcus $\mathrm{Y}$, Rotman-Pikielny $\mathrm{P}$, Benbassat C, Vered I. Vertebral fractures following denosumab discontinuation in patients with prolonged exposure to bisphosphonates. Calcif Tissue Int. 2018;103:44-49.

10. Sosa Henríquez M, de Tejada G, Romero MJ, Escudero-Socorro M, Torregrosa SO. Hip fractures following denosumab discontinuation: three clinical cases reports. J R Soc Med. 2019;112:472-475.

11. Niimi R, Kono T, Nishihara A, Hasegawa M, Kono T, Sudo A. Second rebound-associated vertebral fractures after denosumab discontinuation. Arch Osteoporos. 2020;15:10-12.

12. Genant HK, Wu CY, van Kuijk C, Nevitt MC. Vertebral fracture assessment using a semiquantitative technique. J Bone Miner Res. 1993;8:1137-1148.

13. World Medical Association. World Medical Association Declaration of Helsinki. Ethical principles for medical research involving human subjects. JAMA. 2013;310:2013-2016.

14. Fernández E, Benavent Núñez D, Bonilla Hernán G, et al. Fracturas vertebrales múltiples tras la suspensión de tratamiento con denosumab: serie de diez casos. Reumatol Clínica. 2020;16:480-484.

15. Florez H, Ramírez J, Monegal A, Guañabens N, Peris P. Spontaneous vertebral fractures after denosumab discontinuation: a case collection and review of the literature. Semin Arthritis Rheum. 2019;49:197-203.

16. Tripto-Shkolnik L, Fund N, Rouach V, Chodick G, Shalev V, Goldshtein I. Fracture incidence after denosumab discontinuation: real-world data from a large healthcare provider. Bone. 2020;130:115150.

17. Iranikhah M, Deas C, Murphy P, Freeman MK. Effects of denosumab after treatment discontinuation: a review of the literature. Consult Pharm. 2018;33:142-151.

18. Tsourdi E, Langdahl B, Cohen-Solal M, et al. Discontinuation of Denosumab therapy for osteoporosis: a systematic review and position statement by ECTS. Bone. 2017;105:11-17.

19. Aubry-Rozier B, Gonzalez-Rodriguez E, Stoll D, Lamy O. Severe spontaneous vertebral fractures after denosumab discontinuation: three case reports. Osteoporos Int. 2016;27:1923-1925.

20. Anastasilakis AD, Makras P. Multiple clinical vertebral fractures following denosumab discontinuation. Osteoporos Int. 2016;27:1929-1930.
21. Rao SD, Qiu S, Dhaliwal R, Bhadada SK. Letter to the editor: Severe rebound-associated vertebral fractures after denosumab discontinuation. J Clin Endocrinol Metab. 2017;102:2111.

22. Niimi R, Kono T, Nishihara A, Hasegawa M, Kono T, Sudo A. Reboundassociated vertebral fractures after discontinuation of denosumab for the treatment of maxillitis. Osteoporos Int. 2018;29:769-772.

23. Díez-Pérez A. The debate over the FRAX scale. Rev Osteoporos Metab Miner. 2010;2:5-6.

24. Kanis JA, Harvey NC, Cooper C, Johansson H, Odén A, McCloskey EV. A systematic review of intervention thresholds based on FRAX: A report prepared for the National Osteoporosis Guideline Group and the International Osteoporosis Foundation. Arch Osteoporos. 2016;11:25.

25. Kanis JA, McCloskey EV, Johansson H, Oden A, Ström O, Borgström F. Development and use of FRAX® in osteoporosis. Osteoporos Int. 2010;21:407-413.

26. Tosteson ANA, lii LJM, Dawson-Hughes B, et al. Cost-effective osteoporosis treatment thresholds: the United States perspective from the national osteoporosis foundation guide committee. Osteoporos Int. 2009;19:437-447.

27. Lewiecki EM, Cummings SR, Cosman F. Treat-to-target for osteoporosis: Is now the time? J Clin Endocrinol Metab. 2013;98:946-953.

28. Garber AJ. Treat-to-target trials: uses, interpretation and review of concepts. Diabetes, Obes Metab. 2014;16:193-205.

29. Nogués $X$, Nolla JM, Casado $E$, et al. Spanish consensus on treat to target for osteoporosis. Osteoporos Int. 2018;29:489-499.

30. McClung MR. Cancel the denosumab holiday. Osteoporos Int. 2016;27:1677-1682.

31. Sosa Henríquez M, de Tejada G, Romero MJ, Malouf SJ. Clinical case to debate: therapeutic holidays: yes or no? Rev Osteoporos Metab Miner. 2014;6:63-69.

32. Khan AA, Morrison A, Hanley DA, et al. Diagnosis and management of osteonecrosis of the jaw: A systematic review and international consensus. J Bone Miner Res. 2015;30:3-23.

33. Ramírez L, López-Pintor RM, Casañas E, de Arriba L, Hernández G. New non-bisphosphonate drugs that produce osteonecrosis of the jaws. Oral Health Prev Dent. 2015;13:385-393.

34. Sosa-Henríquez M, Gómez de Tejada-Romero MJ, Bagán-Sebastián $\mathrm{JV}$, et al. Osteonecrosis of the jaw. Rev Osteoporos Metab Miner. 2009;1:53-61.

35. Zanchetta MB, Boailchuk J, Massari F, et al. Discontinuation of denosumab and associated fracture incidence: analysis from the fracture reduction evaluation of denosumab in osteoporosis every 6 months (FREEDOM) trial. J Bone Miner Res. 2013;28:805-807.

36. Eastell R, Christiansen C, Grauer A, et al. Effects of denosumab on bone turnover markers in postmenopausal osteoporosis. J Bone Miner Res. 2011;26:530-537.

How to cite this article: Sosa-Henríquez $\mathrm{M}$, Torregrosa $\mathrm{O}$, Déniz $A$, et al. Multiple vertebral fractures after suspension of denosumab. A series of 56 cases. Int J Clin Pract. 2021;00:e14550. https://doi.org/10.1111/ijcp.14550 